\title{
Observações e modelos - O trabalho de Hodgkin e Huxley como exemplo
}

\author{
Tainara M. A. Soares* \\ Graduanda em Ciências Biológicas \\ UENF/CEDERJ, Petrópolis, RJ \\ E-mail: tainara@lncc.br
}

\author{
Maurício Vieira Kritz \\ Departamento de Matemática Aplicada e Computacional \\ LNCC, Petrópolis, RJ \\ E-mail: kritz@1ncc.br
}

\begin{abstract}
O objetivo deste trabalho é fazer uma revisão do trabalho de Hodgkin e Huxley como um exercício de aprendizado do método científico, de modelagem matemática, bem como da relação entre modelos e observações. O modelo se refere à propagação de pulsos elétricos em axônios e foi desenvolvido com o intuito de entender a capacidade dos neurônios em gerar pulsos elétricos. O experimento empregava o axônio gigante da lula do gênero Loligo, em um método que consistia no disparo de uma corrente elétrica em um ponto do axônio e na observação do efeito dessa corrente na excitabilidade da membrana em outro ponto. Revelando que a membrana é submetida a um aumento de condutância a qual esta tem o mesmo padrão no tempo que a mudança elétrica. $\mathrm{O}$ experimento não mostrava quais íons estavam envolvidos, porém consistiu em uma forte evidência de que ocorria um aumento da permeabilidade iônica. Com a evolução do experimento descobriu-se que o potencial de ação apresentava valores frequentemente maiores que o potencial de repouso, tendo sido estabelecido o valor absoluto dos potenciais naturais de membrana quando o axônio está em repouso. As equações que descrevem o modelo foram construídas registrando-se as correntes e analisando seus gráficos. Em decorrência deste modelo, foram descobertos alguns fenômenos da membrana neuronal, o que levou ao surgimento da neurociência computacional. Ainda hoje surgem novas perspectivas a partir do trabalho de Hodgkin e Huxley, que sem tecnologias e aparelhagens suficientes, já prediziam na época a existência dos canais iônicos e outros mecanismos importantes para o entendimento da natureza dos pulsos elétricos e de sua propagação. Este trabalho está publicado como Relatório Técnico no 01/2014 do Departamento de Matemática Aplicada e Computacional no site do LNCC de onde foram extraídas as referências abaixo.
\end{abstract}

Palavras-chave: Membrana Neuronal, Canais Iônicos, Modelos Matemáticos, Hodgkin e Huxley 


\section{Referências}

[1] H. Alle; A. Roth e J. Geigen, Energy-Efficient Action Potentials in Hippocampal Mossy Fiber. Science 325, 1405 (2009).

[2] R. Fitzhugh, Impulses and physiological states in models of nerve membrane, Biophys. J., 1:445-466. (1961)

[3] Gerstner e Kistler, Spiking Neuron Models. Single Neurons, Populations, Plasticity. Cambridge University Press, 2002. Disponível em: http://icwww.epfl.ch/ gerstner/SPNM/node21.html Acesso em: 01/10/2013.

[4] A. F. Huxley, The quantitative analysis of excitation and conduction in nerve. Nobel Lecture, p. 52-69, (1963). Disponível em : http://www.nobelprize.org/nobel_prizes/medicine/laureates/1963/huxleylecture.pdf. Acesso em 02/11/2012.

[5] A. L. Hodgkin, A. F. Huxley, Currents carried by sodium and potassium ions through the membrane of the giant axon of Loligo. J. Physiol., v. 116, n. 4, p. 449472, (1952). Disponível em: http://www.ncbi.nlm.nih.gov/pmc/articles/PMC1392213/Acesso em 02/11/2012

[6] A. L. Hodgkin e A. F. Huxley, A quantitative description of membrane current and its application to conduction and excitation in nerve. Bulletin of Mathematical Biology. v. 52, n. 1/2, p. 25-71, (1990). Disponível em: http://www.springerlink.com/content/64v078t42g737k42/ Acesso em 02/11/2012

[7] R. Lent, "Cem bilhões de neurônios: conceitos fundamentais de neurociência", Atheneu, São Paulo, 2005.

[8] C. Morris e H. Lecar, Voltage oscillations in the barnacle giant muscle fiber. Biophys. J., pages 193-213, (1981).

[9] J. S. Nagumo, S. Arimoto, e S.Yoshizawa, An active pulse transmission line simulating nerve axon. Proc. IRE, 50:2061-2070, (1962).

[10] 1/2014; T. M. A. Soares e M. V. Kritz, Observações e modelos - O trabalho de Hodgkin e Huxley como exemplo. Relatório Técnico, LNCC, Petrópolis/RJ, (2014). 